\title{
Local critical stress correlates better than global maximum stress with plaque morphological features linked to atherosclerotic plaque vulnerability: an in vivo multi-patient study
} Dalin Tang*1, Zhongzhao Teng1, Gador Canton², Thomas S Hatsukami³, Li Dong ${ }^{2}$, Xueying Huang ${ }^{1}$ and Chun Yuan ${ }^{2}$

Address: ${ }^{1}$ Worcester Polytechnic Institute, Mathematical Sciences Department 100 Institute Road, Worcester, MA, USA, ${ }^{2}$ University of Washington, Department of Radiology, Seattle, WA 98195 USA and ${ }^{3}$ University of Washington, Division of Vascular Surgery, Seattle, WA 98195 USA

Email: Dalin Tang* - dtang@wpi.edu; Zhongzhao Teng - zzteng@wpi.edu; Gador Canton - gcanton@u.washington.edu; Thomas S Hatsukami - tomhat@u.washington.edu; Li Dong - lidong@u.washington.edu; Xueying Huang - xueying@wpi.edu; Chun Yuan - cyuan@u.washington.edu

* Corresponding author

Published: 3 August 2009

BioMedical Engineering OnLine 2009, 8:15 doi:10.1186/1475-925X-8-15
Received: 30 May 2009

Accepted: 3 August 2009

This article is available from: http://www.biomedical-engineering-online.com/content/8/I/I5

(C) 2009 Tang et al; licensee BioMed Central Ltd.

This is an Open Access article distributed under the terms of the Creative Commons Attribution License (http://creativecommons.org/licenses/by/2.0), which permits unrestricted use, distribution, and reproduction in any medium, provided the original work is properly cited.

\begin{abstract}
Background: It is believed that mechanical stresses play an important role in atherosclerotic plaque rupture process and may be used for better plaque vulnerability assessment and rupture risk predictions. Image-based plaque models have been introduced in recent years to perform mechanical stress analysis and identify critical stress indicators which may be linked to rupture risk. However, large-scale studies based on in vivo patient data combining mechanical stress analysis, plaque morphology and composition for carotid plaque vulnerability assessment are lacking in the current literature.
\end{abstract}

Methods: 206 slices of in vivo magnetic resonance image (MRI) of carotid atherosclerotic plaques from 20 patients (age: 49-7I, mean: 67.4; all male) were acquired for model construction. Modified Mooney-Rivlin models were used for vessel wall and all plaque components with parameter values chosen to match available data. A morphological plaque severity index (MPSI) was introduced based on in vivo plaque morphological characteristics known to correlate with plaque vulnerability. Critical stress, defined as the maximum of maximum- principalstress (Stress- $\left.P_{1}\right)$ values from all possible vulnerable sites, was determined for each slice for analysis. A computational plaque stress index (CPSI, with 5 grades $0-4,4$ being most vulnerable) was defined for each slice using its critical stress value and stress interval for each CPSI grade was optimized to reach best agreement with MPSI. Correlations between CPSI and MPSI, plaque cap thickness, and lipid core size were analyzed.

Results: Critical stress values correlated positively with lipid core size $(r=0.3879)$ and negatively with cap thickness $(r=-0.3953)$. CPSI classifications had 71.4\% agreement with MPSI classifications. The Pearson correlation coefficient between CPSI and MPSI was $0.849(\mathrm{P}<0.000 \mathrm{I})$. Using global maximum Stress- $P$, value for each slice to define a global maximum stress-based CPSI (G-CPSI), the agreement rate with MPSI was only $34.0 \%$. The Pearson correlation coefficient between G-CPSI and MPSI was 0.209.

Conclusion: Results from this in vivo study demonstrated that localized critical stress values had much better correlation with plaque morphological features known to be linked to plaque rupture risk, compared to global maximum stress conditions. Critical stress indicators have the potential to improve image-based screening and plaque vulnerability assessment schemes. 


\section{Introduction}

Atherosclerotic plaques may rupture without warning and cause acute cardiovascular syndromes such as heart attack and stroke. Currently, screening and diagnosis of patients with atherosclerotic plaques are based on medical images such as magnetic resonance image (MRI), ultrasound, intravascular ultrasound (IVUS), or computerized tomography (CT). Increasing evidences showed that such imageonly screening and diagnostic techniques are insufficient to identify those victims before the event occurs $[1,2]$. It has been hypothesized that mechanical forces (rupture triggers) play an important role in plaque rupture process and should be considered in an integrated way with plaque morphology and composition for possible improvement of plaque assessment schemes [3]. Largescale studies based on in vivo patient data combining mechanical stress analysis, plaque morphology and compositions are needed to identify the critical stress indicators that are linked to plaque vulnerability.

In recent years, MRI techniques have shown their ability to non-invasively determine plaque size, shape and component [4-6]. Yuan et al. developed multi-contrast techniques to improve the quality of MR-images and to better differentiate various components of the plaque $[4,6]$. Attempts of using ultrasound and IVUS techniques have been made to quantify vessel motion, mechanical properties and vessel wall structure, even to predict rupture locations $[7,8]$. Using non-invasive MRI techniques, Cai et al. developed a classification system for carotid plaques based on in vivo MRI [9]. MRI- and histology-based computational simulations for plaque rupture investigation and vulnerability assessment have been conducted by several groups and many interesting and significant results have been reported [10-24]. Holzapfel et al. introduced multi-layer 3D models for the simulation of balloon angioplasty using MRI and direct mechanical testing [10]. Steinman studied influence of complex vessel geometry on flow behaviors using image-based realistic arterial geometries [11]. Weinbaum et al. investigated critical stress behaviors at plaque cap with micro-calcification inclusions and elevated stress levels were observed [16]. Bluestein et al. introduced fluid-structure interaction models to study influence of microcalcifications on vulnerable plaque mechanics [17]. Li et al. showed that wall stress calculated based on in vivo MRI of carotid arteries was higher in symptomatic patients than in asymptomatic patients [19]. Groen and Wentzel et al. reported a followup case study showing high flow shear stress region was associated with site of plaque rupture [21]. Tang et al. introduced a "local maximum stress hypothesis" to identify the critical site and stress conditions in the plaque and proposed an ex vivo MRI-based computation plaque vulnerability index (CPVI) to access plaque vulnerability [12]. Results from 34 2D ex vivo MRI slices from 14 human atherosclerotic coronary arteries indicated that CPVI has a good agreement (89\%) with histology-based assessment.

Most of the current computational studies were at model development stage using one or a few samples. Large-scale in vivo MRI-based plaque mechanical analysis is lacking in the current literature. Due to the complexity of plaque architecture, analyzing the large amount of computational stress/strain data and identifying critical stress indicators which correlate closely with plaque vulnerability are time-consuming and challenging. A validated easy-touse stress index would be more practical for potential patient screening and clinical applications.

In this paper, we extend our previous ex vivo study to an in vivo MRI-based multi-patient study to further validate that it is the localized critical stress conditions, not global maximum stress conditions, that have better correlations with plaque morphological features known to be linked to plaque vulnerability. A morphological plaque severity index (MPSI) and a computational plaque stress index (CPSI) were introduced for plaque classification and comparison. Correlations between CPSI and MPSI, plaque cap thickness, and lipid core size were quantified.

\section{MRI data, method and model MRI Data Acquisition}

Two hundred and six (206) high resolution in vivo MRI slices of carotid atherosclerotic plaques from 20 patients (age: 49-71, mean age: 67.4; all male) were provided by Vascular Imaging Laboratory of the University of Washington (UW) using protocols approved by UW Institutional Review Board and segmented by a self-developed software package (CASCADE) [25]. Due to limited resources and difficulty in getting healthy volunteers, each 2D slice was treated as an independent case to gain a good representation of all lesion types. Patients were imaged with a 1.5-T MR scanner (Signa Horizon EchoSpeed, General Electric Health Care). Precontrast MR images that included double-inversion-recovery $\mathrm{T} 1 \mathrm{~W}$, proton densityweighted (PDW), T2W, TOF, and postcontrast doubleinversion-recovery T1W MR images of carotid arteries were obtained with a previously published standardized protocol (T1W: repetition time/echo time/inversion time, 800/10/650 ms; PDW and T2W: repetition time/echo time, 3RR, 20/40 ms; TOF: repetition time/echo time, 23/ $3.8 \mathrm{~ms}$ ) [4-6]. Plane and space resolution was at $0.31 \times$ $0.31 \times 2.0 \mathrm{~mm}^{3}$ (with scanner interpolation). Figure 1 gives 4 selected MRI slices with segmented contour plots and corresponding $3 \mathrm{D}$ rendered geometry of the plaque.

\section{Assignment of Morphological Plaque Severity Index (MPSI)}

Since histological data is in general not available for in vivo studies, a morphological plaque severity index 
(a) In Vivo MRI Images
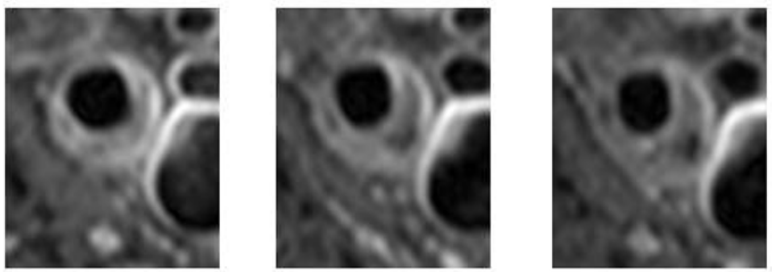

(b) Segmented contonrs

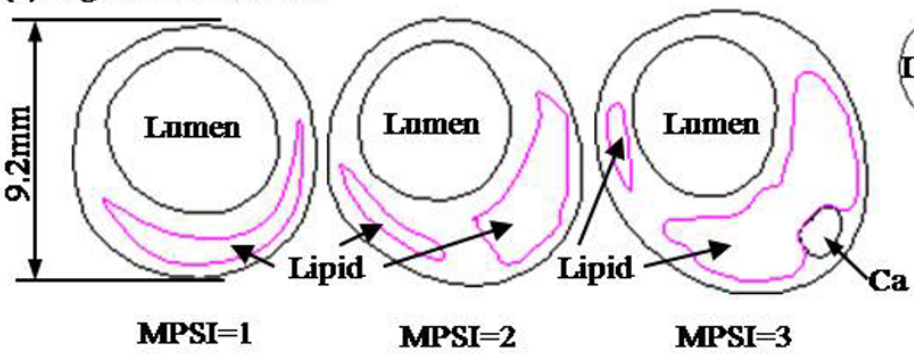

(d) Critical Siteand Critical Stress (KPa) on Stress-P, BandPlot

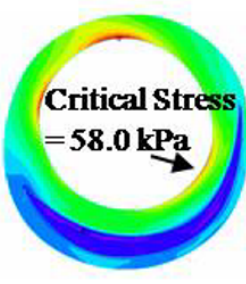

CPVI $=1$

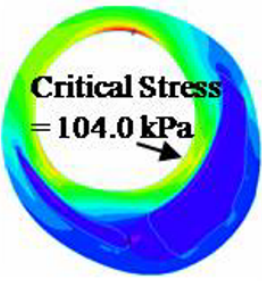

CPVI $=2$

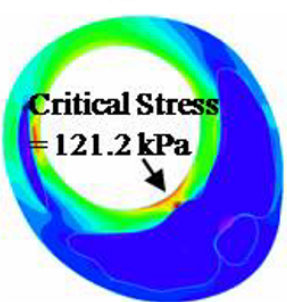

CPVI $=3$

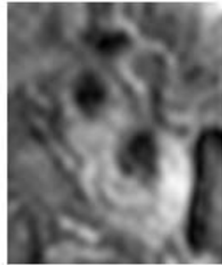

(c) Rendered3D view

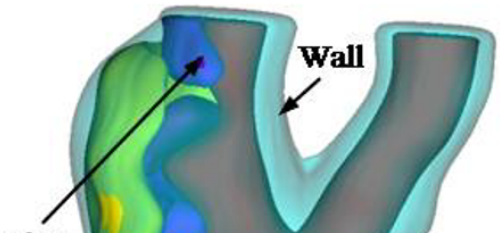

Ulcer

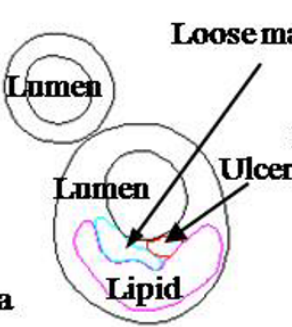

MPSI $=4$

atrix

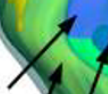

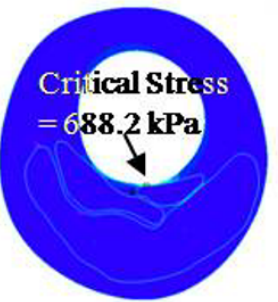

Min Universal Scale Max

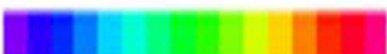

CPVI $=4$

\section{Figure I}

(a) In vivo MR-images (Lumen is marked by a red asterisk); (b) Segmented contour plots showing plaque components (Black: Lumen, vessel wall or calcification; Magenta: Lipid core; Red: Ulcer); (c): Rendered 3D view using the segmented contours; (d): Band plot of maximal principal stress (Stress- $P_{I}$ ) of corresponding slices; CPSI values were calculated based on critical Stress- $P_{1}$ values at critical sites, following procedures described in the paper.

(MPSI) was introduced (Table 1) and assigned to each segmented MRI slice based on plaque morphological features known to correlate with plaque vulnerability from histopathological studies $[3,9,12]$. These features include: 1 ) the size and distribution of the soft lipid rich necrotic core (LRNC); 2) the fibrous cap thickness (which correlates with plaque stability); and 3 ) the presence of ulcer, intraplaque hemorrhage and thrombi. MPSI values $(0,1,2,3$ to 4 ) indicate the level of increasing severity. The MPSI definitions are closely associated with the AHA (American Heart Association) lesion type classifications (see Table 1) and the representative morphologies are shown in Figure 1 (b).

Introduction of MPSI provides a combined morphological index for plaque classification. This is similar to the histopathological plaque vulnerability index (HPVI) previously proposed in our ex vivo study for the vulnerability of coronary plaque [12]. The semi-quantitative MPSI will serve as a "benchmark" to validate the CPSI in this paper. MPSI distributions of the 206 slices are listed in Table 2.

\section{A Pre-Shrink Process for In Vivo Data}

The In vivo imaged arteries were under physiological pressure conditions. Therefore a pre-shrink process was necessary to obtain the zero-pressure geometry, which was used as the numerical starting geometry, and to recover the in vivo geometry when pressure was imposed in the lumen. In this study, for each patient, the shrinkage was determined by choosing the most circular (round) slice which would recover its in vivo shape best when lumen pressure was imposed. The shrinkage rates of contours of lumen $\left(\delta_{\text {in }}\right)$ and outer wall $\left(\delta_{\text {out }}\right)$ were numerically determined following an iterative procedure so that: 1) the vessel cross-section area was conserved (conservation of mass); and 2) the pressurized morphology and the original in vivo morphology had the best agreement. The determined shrinkage was applied to all the slices of this patient for consistency and uniformity and for future comparison 
Table I: Morphological plaque vulnerability index (MPSI) classifications and comparison with AHA classifications

\begin{tabular}{|c|c|c|c|}
\hline MPSI Category & $\begin{array}{l}\text { Corresponding AHA lesion types } \\
\text { (modified) }\end{array}$ & Description & Level of vulnerability \\
\hline 0 & | or II & Normal or nearly normal wall. & Very stable \\
\hline I & III & $\begin{array}{l}\text { Moderate intimal thickening, no extracellular lipid, } \\
\text { calcification or significant inflammation. }\end{array}$ & Stable \\
\hline 2 & $\begin{array}{l}\text { IV } / \mathrm{V} \text { with less than } 30 \% \mathrm{NC} \text { by area; or } \\
\text { VII; or VIII }\end{array}$ & $\begin{array}{l}\text { Advanced lesion with small necrotic core ( }<30 \% \text { of plaque } \\
\text { size), or can be fibrotic or calcified, thick fibrous cap }(>200 \\
\mu \mathrm{m}) \text {. }\end{array}$ & Slightly unstable \\
\hline 3 & IV/V with $30-40 \%$ NC by area & $\begin{array}{l}\text { Advanced lesion with Moderate lipid core }(30-40 \% \text { of } \\
\text { plaque size) and fibrous cap }(150-200 \mu \mathrm{m}) \text {. }\end{array}$ & Moderately unstable \\
\hline 4 & IV $/ \mathrm{V}$ with $>40 \% \mathrm{NC}$ by area; or $\mathrm{VI}$ & $\begin{array}{l}\text { Advanced lesion with a very large necrotic core }(>40 \%) \text {, } \\
\text { thin fibrous cap }(<150 \mu \mathrm{m}) \text {, or with fibrous cap rupture, } \\
\text { ulceration, or intraplaque hemorrhage. }\end{array}$ & Very unstable \\
\hline
\end{tabular}

Quantitative plaque lipid core size and cap thickness information will be based on plaque component contour information in this paper. In particular, plaque cap thickness will be calculated as the shortest distance between a lipid core and lumen, while the thin plaque cap may not be directly measurable by MRI.

with 3D models. This approach was chosen also because slice-specific shrinkage data would not be available in patient-screening practice. The average inner circumference shrinkage rate $\left(\delta_{\text {in }}\right)$ was $12 \%(S D \pm 2.2 \%)$ for the 206 2D models.

Figure 2 gives an example which shows that maximum principal stress (Stress- $\mathrm{P}_{1}$ ) would be over-estimated by $21 \%$ if pre-shrink process was not performed. The pressurized vessel dimension with pre-shrink (Figure 2(d)) matched in vivo dimension (error $<0.4 \%$ ). Without preshrink, vessel dimension expanded about 10\% (Figure 2(c)).

\section{Mesh Generation, Computational Models and Solution Methods}

Model mesh generation, solution and data post-processing were performed using ADINA, a commercial finite element package (ADINA R\&D, Inc., Watertown, MA), which has been used by Tang et al. in recent years to solve athero- sclerotic artery models $[12-15,24]$. The computational mesh was made using ADINA automated mesh generation system. Finer meshes were used for thin-cap and large curvature areas to avoid computational artifacts. A typical plaque sample with 4 slices was given by Figure 1, with dimension indicated on one slice. The solid models are given below:

$$
\begin{gathered}
\rho \mathrm{v}_{\mathrm{i}, \mathrm{tt}}=\sigma_{\mathrm{ij}, \mathrm{j}}, \mathrm{i}, \mathrm{j}=1,2,3 \text {; sum over } \mathrm{j}, \\
\varepsilon_{\mathrm{ij}}=\left(\mathrm{v}_{\mathrm{i}, \mathrm{j}}+\mathrm{v}_{\mathrm{j}, \mathrm{i}}+\mathrm{v}_{\alpha, \mathrm{i}} \mathrm{v}_{\alpha, \mathrm{j}}\right) / 2, \mathrm{i}, \mathrm{j}, \alpha=1,2,3, \\
\left.\sigma_{\mathrm{ij}} \cdot \mathrm{n}_{\mathrm{j}}\right|_{\text {out_wall }}=0, \\
\left.\sigma_{\mathrm{ij}}^{\mathrm{r}} \cdot \mathrm{n}_{\mathrm{j}}\right|_{\text {interface }}=\left.\sigma_{\mathrm{ij}}^{\mathrm{s}} \cdot \mathrm{n}_{\mathrm{j}}\right|_{\text {interface }}
\end{gathered}
$$

where $\sigma$ is stress tensor (superscripts indicate different materials), $\varepsilon$ is strain tensor, $\mathbf{v}$ is solid displacement vec-

Table 2: Case distributions according to MPSI and agreement rate between CPSI and MPSI

\begin{tabular}{cccc}
\hline MPSI & Number of Slices & Percentage (\%) & Agreement Rate (\%) \\
\hline 0 & 33 & 16.02 & 64.44 \\
\hline 1 & 45 & 21.84 & 57.14 \\
\hline 3 & 42 & 20.39 & 57.50 \\
\hline 4 & 40 & 19.42 & 82.61 \\
\hline
\end{tabular}


(a) Original In Vivo MRI Data

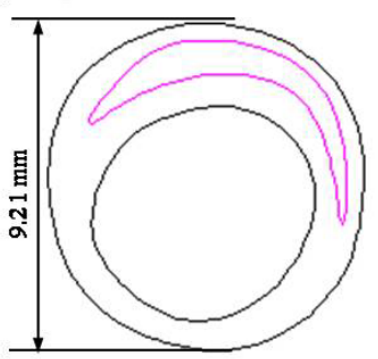

(c) Stress- $P_{1}$ Obtained from (a).

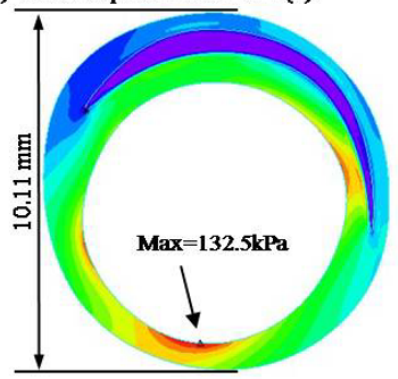

(b) Contours with Pre-Shrink

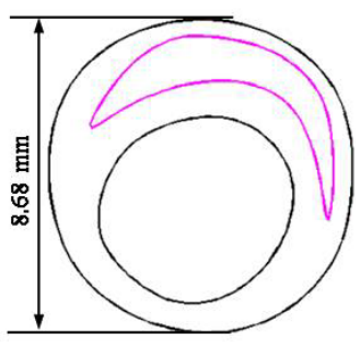

(d) Stress- $\mathbf{P}_{1}$ Obtained from (b).

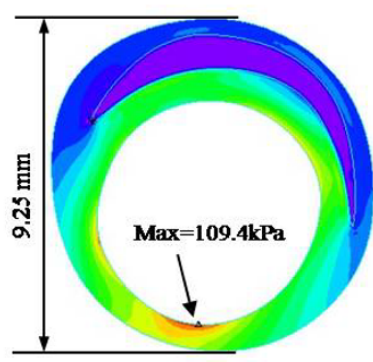

Figure 2

Geometry and Stress-P, plots of one example showing that the pre-shrink process improves stress predictions. Patient pressure: Pmean $=121.5 \mathrm{mmHg}$. Shrinkage: Inner wall: I4\%; Outer wall: 5.8\%. Maximum Stress- $P_{\text {, over- }}$ estimation without pre-shrink: $21 \%$.

tor, superscript letters " $r$ " and " $s$ " were used to indicate different materials. No-slip conditions and natural traction equilibrium conditions are assumed at all interfaces. For simplicity, all material densities were set to $1 \mathrm{~g} \cdot \mathrm{cm}^{-3}$ in this paper.

All plaque components including fibrous tissue, hemorrhage, lipid core, calcification and loose matrix were assumed to be non-linear, isotropic and hyper-elastic. The modified Mooney-Rivlin model was used as the material model [26]:

$$
W=c_{1}\left(I_{1}-3\right)+c_{2}\left(I_{2}-3\right)+D_{1}\left[\exp \left(D_{2}\left(I_{1}-3\right)\right)-1\right]
$$

$$
I_{1}=\sum C_{i i}, I_{2}=1 / 2\left[I_{1}^{2}-C_{i j} C_{i j}\right]
$$

where $I_{1}$ and $I_{2}$ are the first and second invariants, $\mathrm{C}=\left[\mathrm{C}_{\mathrm{ij}}\right]$ $=\mathbf{X}^{\mathrm{T}} \mathbf{X}$ is the right Cauchy-Green deformation tensor, $\mathbf{X}=$ $\left[\mathrm{X}_{\mathrm{ij}}\right]=\left[\partial \mathrm{x}_{\mathrm{i}} / \partial \mathrm{a}_{\mathrm{j}}\right],\left(\mathrm{x}_{\mathrm{i}}\right)$ is the current position, $\left(\mathrm{a}_{\mathrm{i}}\right)$ is the original position. Material parameters $c_{i}$ and $D_{i}(i=1,2)$ were chosen to match available experimental measurement data [12,14,23]: vessel material/fibrous cap, $c_{1}=36.8 \mathrm{kPa}$, $D_{1}=14.4 \mathrm{kPa}, D_{2}=2$; lipid core/hemorrhage, $c_{1}=2 \mathrm{kPa}$, $D_{1}=2 \mathrm{kPa}, D_{2}=1.5$; calcification, $\mathrm{c}_{1}=368 \mathrm{kPa}, \mathrm{D}_{1}=144$ $\mathrm{kPa}, \mathrm{D}_{2}=2.0$; loose matrix, $c_{1}=18.4 \mathrm{kPa}, D_{1}=7.2 \mathrm{kPa} ; D_{2}$

= 1.5. $c_{2}=0$ for all materials. A pulsating pressure was imposed in the lumen using the systolic/diastolic arm pressure data for each patient from their last hospital admission. Average systolic and diastolic pressures were $144.7 \pm 21.5$ and $75.6 \pm 13.5 \mathrm{mmHg}$ respectively, for the 20 patients studied. Pressure at the out-boundary of each vessel slice was set to zero.

The computational plaque models were solved using ADINA which uses unstructured finite element methods, nonlinear incremental iterative procedures and the Newton-Raphson iteration method to deal with complex geometries and nonlinear systems. Details of the models and methods are given in Tang et al. [13] and Bathe [26].

\section{Definition and Calculation of Critical Stress}

Critical stress was defined as the maximum of all local maximum Stress- $\mathrm{P}_{1}$ values from possible vulnerable sites. It is known that thin plaque cap is closely related to plaque rupture. Thus vulnerable sites include all locations where a thin region covers a plaque component and a local maximum Stress- $P_{1}$ was found. It should be noted that our "thin region" includes fibrous cap over a lipid core, as well as "cap" over calcification and other plaque components. Healthy sites where no plaque components were present and rupture was unlikely were excluded from the "vulnerable site list", even if a local stress maximum occurred there. For each slice, Stress- $\mathrm{P}_{1}$ distribution corresponding to peak pressure condition was obtained from the $2 \mathrm{D}$ computational model. An automatic search was performed to find all local maximum Stress- $\mathrm{P}_{1}$ values from vulnerable sites. Then critical stress for that slice was determined using the definition given above. The site where critical stress was found was defined as the critical site. For slices without any components, critical stress was set to zero since these slices are assumed to be very stable.

\section{CPSI Assignment, Quantitative Definition of Morphological Features and Data Analysis}

Each slice was assigned a CPSI value $(0,1,2,3$ or 4$)$ according to its critical stress by using five stress intervals, which were determined to have best match rate with MPSI. Cap thickness was defined as the shortest distance between the critical site and the nearest plaque component contour. Normalized lipid index (NLI) and normalized wall index (NWI, also called plaque burden) are defined as:

Normalized lipid index $=$ Area of lipid - rich necrotic core $/$ vessel area,

Normalized wall index (plaque burden $)=($ wall area $) /$ total vessel area.

Correlations between CPSI values and MPSI, plaque cap thickness, normalized lipid index and normalized wall 
index were analyzed. Global maximum Stress- $\mathrm{P}_{1}$ values for each slice were also recorded for comparison and correlation analyses.

\section{Results \\ Correlations between Critical Stress and Cap Thickness, Normalized Lipid Index and Plaque Burden}

Excluding slices without any plaque components and slices with calcification component only, we have 152 slices for this analysis. All morphological quantities were calculated based on segmented MRI data with interpolations as needed in the modeling process. Figure 3 gives plots of critical stresses vs. plaque cap thickness, NLI, and NWI, respectively. Critical stress values correlated with normalized lipid index positively $(\mathrm{r}=0.3879)$ and correlated with cap thickness negatively $(r=-0.3953)$. No correlation was found between critical stress values and NWI (Pearson correlation coefficient $\mathrm{r}=0.0444$ ).

\section{Correlation between CPSI and MPSI}

A simple numerical code was used to determine five equal stress intervals $[0, a),[a, 2 a),[2 a, 3 a),[3 a, 4 a)$, and $[4 a$, +8 ) corresponding to CPSI values $0-4$ to reach the best agreement between CPSI and MPSI. The five intervals (unit: KPa) [0, 35.5), [35.5, 71), [71, 106.5), [106.5, 142), and $[142,+8)$ were used for CPSI values of $0,1,2,3$ and 4 , respectively. The optimized agreement rate was $71.4 \%$ (147 matching cases out of 206). The Pearson correlation coefficient between CPSI and MPSI was 0.849 (p < 0.0001 ). Table 2 lists number of cases and agreement rate for each MPSI grade group. The agreement rate was $82.6 \%$ for the highly vulnerable group (MPSI = 4). According to the CPSI stress intervals, a plaque will be considered highly vulnerable (high risk) if its critical stress is higher than $142 \mathrm{kPa}$.

\section{Correlation between an Index Based on Global Maximum Stress-P, Values and MPSI}

Using global maximum Stress- $\mathrm{P}_{1}$ value for each slice to calculate a global maximum stress-based CPSI (G-CPSI), the agreement rate between G-CPSI and MPSI was 34.0\%. And the Pearson correlation coefficient was 0.209. Figure 4 shows that critical stress values correlate much better with MPSI compared to global maximum Stress- $P_{1}$ values. This is not surprising because global maximal stress often appears at healthy parts of the vessel where either the vessel wall is thinner than the diseased plaque side or the vessel curvature is large, and is not a good indicator for plaque vulnerability assessment [12].

\section{Discussion \\ Purpose of Introducing Localized Critical Stress Indicator and CPSI}

The purpose of introducing the localized critical stress indicator and CPSI index is to identify stress indicators which are more closely linked to plaque vulnerability, compared to global maximum stress conditions. The critical-stress based CPSI provides a plaque classification and assessment scheme which includes mechanical factors, plaque morphological features and tissue compositions for possible patient-screening applications. A 5-point scale (CPSI $=0,1,2,3$, and 4 ) was used so that the CPSIbased classifications would be comparable with AHA plaque classification scheme (Table 1). One main potential advantage for introducing CPSI is that it can provide quantitative assessment of plaques, compared to the current qualitative AHA scheme. More refined scales using decimals may be introduced to provide more quantitative assessment. Results from 206 slices suggested that CPSI and MPSI had a good agreement on plaque classification. Global maximum stress values showed much weaker correlation with MPSI, suggesting that localized critical stress may be a better indicator for plaque vulnerability.

The disagreement $(28.6 \%$ overall, $17.4 \%$ for high risk plaques) suggested that CPSI scheme may complement image-only assessment schemes and lead to potential improvements. The present study is the first large-scale ( $\mathrm{n}$ = 206) case study quantifying differences between mechanics-image combined and morphology-only assessment schemes. In Table 2, the worst match between CPSI and MPSI is in the scale of 1-3. This range of plaque represents the moderate stenosis, but with high risk for future plaque rupture. In fact, this class of plaque is the most interesting plaque that is often ignored in clinical practices. Developing some biomarker to identify the risk of these plaques would be great benefit for clinicians. CPSI could serve as such a biomarker providing additional mechanical stress information for image-based plaque assessment schemes.

It should be understood that plaque rupture is a multi-faceted process. CPSI covers only mechanical and morphological factors. We hope CPSI could provide complementing information for plaque assessment that image alone could not provide. Multiple biomarkers from different channels such as cell activity, lumen surface condition and inflammation should be jointly considered for more complete and accurate vulnerability assessment.

\section{D vs. 3D Models and Model limitation}

We used 2D models in the evaluation of CPSI for commercialization and clinical application purposes. With current modeling and computing power, only $2 \mathrm{D}$ modeling and mechanical analysis are practical. It takes only a few minutes with our automated procedure to make one 2D model, while it takes several weeks for an experienced researcher to construct a 3D fluid-structure interaction (FSI) model which is impractical for clinical applications. 2D and 3D models were compared in our previous studies and it was found that 2D models could provide good approximations to 3D models in correlation and classifi- 
(a) Critical Stress Vs. Cap Thickness

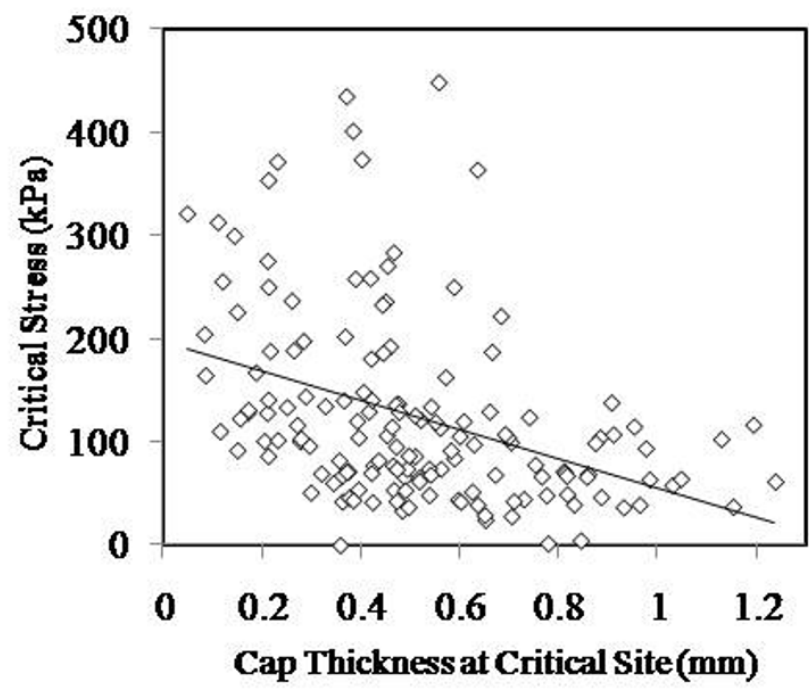

(c) Critical Stress Vs. Normalized Wall Index

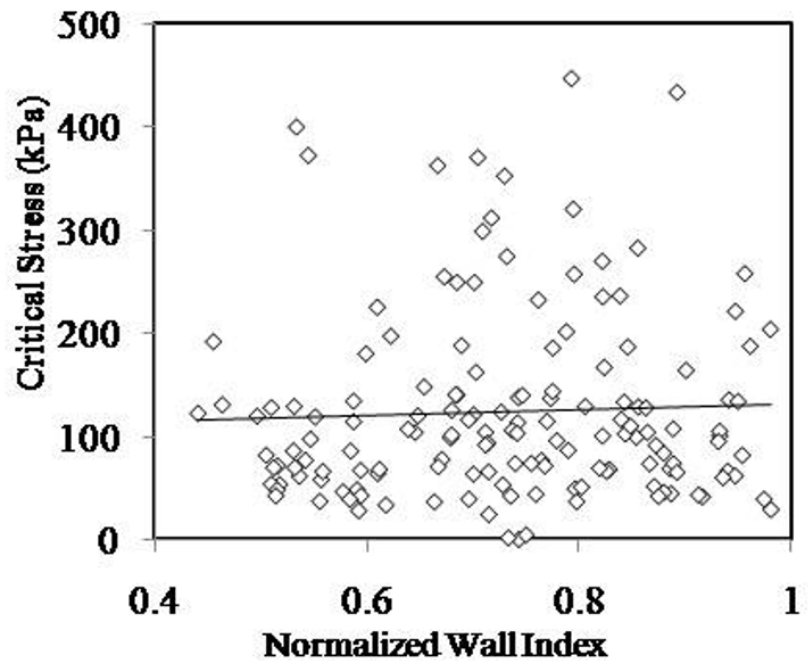

(b) Critical Stress Vs. NormalizedLipidIndex

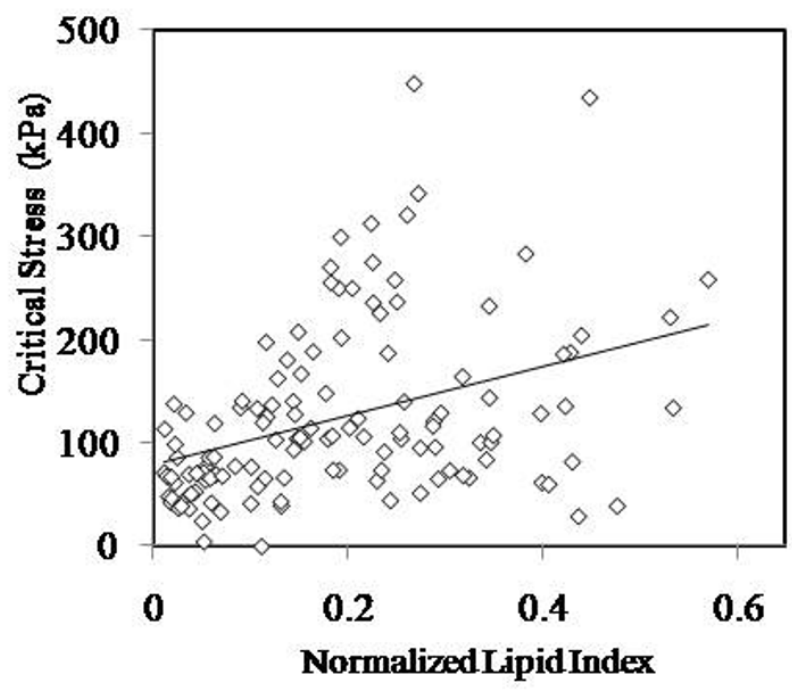

Figure 3

Critical stress values correlate much better with MPSI than global maximum values of Stress-P, from 206 carotid 2D plaque samples (in vivo MRI).

cation studies [13]. While 2D and 3D models do give different Stress- $\mathrm{P}_{1}$ values, Figure 5 shows that they have similar distribution patterns and the majority of those values differ in a proportional way. Stress values from 2D and $3 \mathrm{D}$ plaque models can differ for the following reasons: a) 3D model includes longitudinal stretch which leads to overall (3D mean) increased $3 \mathrm{D}$ Stress- $\mathrm{P}_{1}$ values; b) At the same time, 2D model expands more in radial direction when pressurized because it has no axial stretch and no bonding effect from neighboring slices. That leads to higher Stress- $\mathrm{P}_{1}$ value at inner wall (lumen) and lower
Stress- $\mathrm{P}_{1}$ values at outer portion of the wall as shown by Figure 5c;) 3D longitudinal curvature of the vessel can lead to greater 2D/3D stress prediction differences.

Several other limitations exist in this study: a) Patient-specific material properties were not included because data was not available with current technology; b) some important factors such as lumen surface inflammation and erosion conditions were not taken into account since current in vivo MRI technology could not accurately provide these data; c) residual stress (opening angle) was not 

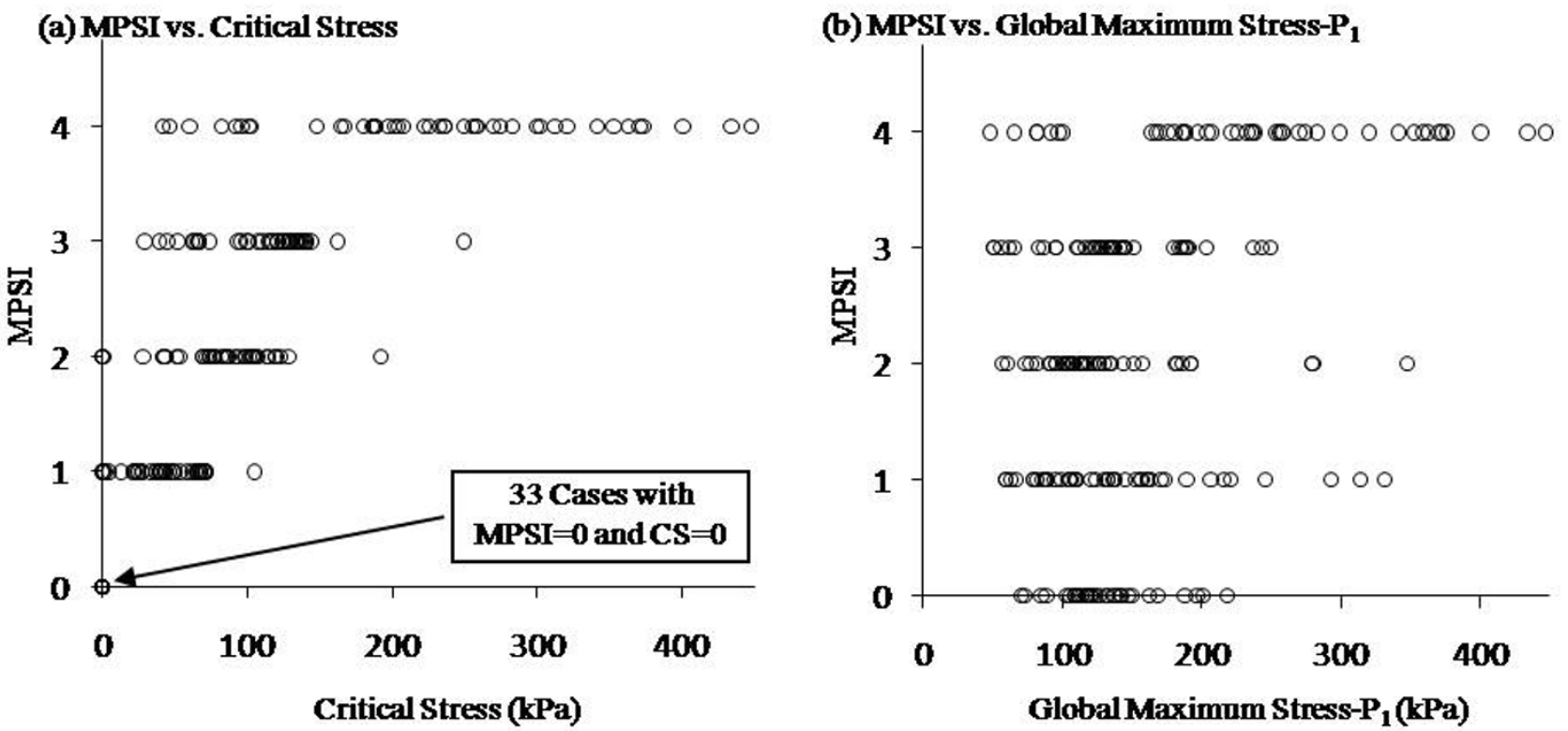

Figure 4

Critical stress values correlated with cap thickness at critical site negatively (Pearson correlation coefficient $r$ $=\mathbf{0 . 3 9 5 3})$ and normalized lipid index positively $(r=\mathbf{0 . 3 8 7 9})$. No correlation between critical stresses and the normalized wall index was found $(r=0.0444)$.

included in our model; d) the CPSI cannot well estimate the risk of cases with very narrow lumen and thick fibrous cap but having large lipid core and other components. Accuracy of CPSI could be improved with accurate cap thickness and material property measurements.

\section{Conclusion}

Results from this multi-patient in vivo study based on 206 slices demonstrated that localized critical stress values had much better correlation with plaque morphological features known to be linked to plaque rupture risk ( $\mathrm{r}=0.849$ with MPSI), compared to global maximum stress condi-

(a) Stress- $P_{1}$ from 3DFSIModel. (b) Stress- $P_{1}$ from 2DModel.
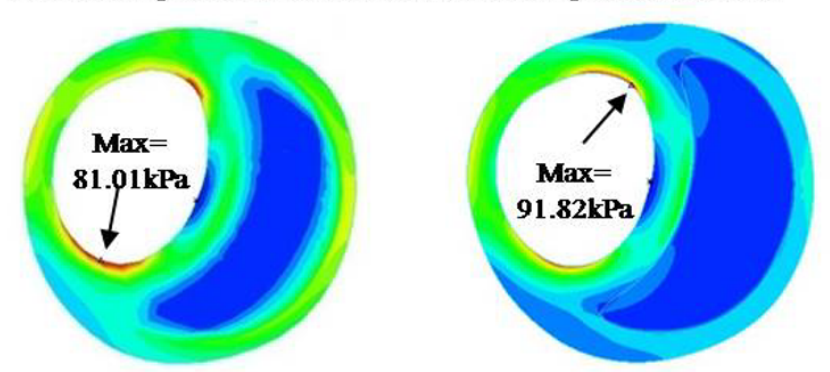

Figure 5

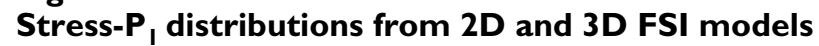
have similar patterns with $2 D$ model giving higher maximum Stress- $P_{\text {, }}$ on the lumen. tions ( $\mathrm{r}=0.209$ with MPSI). Critical stress values correlated positively with normalized lipid index $(r=0.3879)$ and negatively with cap thickness $(r=-0.3953)$. Large scale and long-term patient studies using 3D models are needed to further validate our findings and identify potential stress risk indicators for patient screening plaque vulnerability assessment.

\section{Competing interests}

Other than the grants listed in the acknowledgement section, the authors declare that they have no other competing interest.

\section{Authors' contributions}

DT, ZT, and XH were responsible for computational modeling and data analysis part. CY, GC, TSH, and LD were responsible for MRI data acquisition and segmentation part. All authors 1) have made substantial contributions to conception and design, or acquisition of data, or analysis and interpretation of data; 2) have been involved in drafting the manuscript or revising it critically for important intellectual content; and 3) have given final approval of the version to be published. Each author has participated sufficiently in the work to take public responsibility for appropriate portions of the content.

\section{Authors' information}

Tang's group has been publishing image-based modeling work in recent years. For more information, please visit 
Tang's website: http://users.wpi.edu/ dtang/. Dr. Yuan's group and their lab (Vascular Imaging Laboratory, University of Washington) have been developing MR imaging methods and have published extensively in this area. For more information, please visit their website: http:// www.rad.washington.edu/research/our-research/groups/ vil.

\section{Acknowledgements}

This research was supported in part by NSF grant DMS-0540684 and NIH grant RO I EB004759. Many helpful discussions with Dr. William Kerwin and Ms. Marina Ferguson are happily acknowledged.

\section{References}

I. Naghavi M, Libby P, Falk E, Casscells SW, Litovsky S, Rumberger J, Badimon J], Stefanadis C, Moreno P, Pasterkamp G, Fayad Z, Stone PH, Waxman S, Raggi P, Madjid M, Zarrabi A, Burke A, Yuan C, Fitzgerald PJ, Siscovick DS, de Korte CL, Aikawa M, Juhani Airaksinen $\mathrm{KE}$, Assmann G, Becker CR, Chesebro JH, Farb A, Galis ZS, Jackson C, Jang IK, Koenig W, Lodder RA, March K, Demirovic J, Navab M, Priori SG, Rekhter MD, Bahr R, Grundy SM, Mehran R, Colombo A, Boerwinkle E, Ballantyne C, Insull W Jr, Schwartz RS, Vogel R, Serruys PW, Hansson GK, Faxon DP, Kaul S, Drexler H, Greenland P, Muller JE, Virmani R, Ridker PM, Zipes DP, Shah PK, Willerson JT: From vulnerable plaque to vulnerable patient: a call for new definitions and risk assessment strategies: Part I. Circulation 2003, 108(14): 1664-72.

2. Naghavi M, Libby P, Falk E, Casscells SW, Litovsky S, Rumberger J, Badimon JJ, Stefanadis C, Moreno P, Pasterkamp G, Fayad Z, Stone $\mathrm{PH}$, Waxman S, Raggi P, Madjid M, Zarrabi A, Burke A, Yuan C, Fitzgerald PJ, Siscovick DS, de Korte CL, Aikawa M, Juhani Airaksinen $\mathrm{KE}$, Assmann G, Becker CR, Chesebro JH, Farb A, Galis ZS, Jackson C, Jang IK, Koenig W, Lodder RA, March K, Demirovic J, Navab M, Priori SG, Rekhter MD, Bahr R, Grundy SM, Mehran R, Colombo A, Boerwinkle E, Ballantyne C, Insull W Jr, Schwartz RS, Vogel R, Serruys PW, Hansson GK, Faxon DP, Kaul S, Drexler H, Greenland P, Muller JE, Virmani R, Ridker PM, Zipes DP, Shah PK, Willerson JT: From vulnerable plaque to vulnerable patient: a call for new definitions and risk assessment strategies: Part II. Circulation 2003, 108(15): 1772-8.

3. Fuster V: The Vulnerable Atherosclerotic Plaque: Understanding, Identification, and Modification Edited by: Fuster V, Cornhill JF, Dinsmore RE, Fallon JT, Insull W, Libby P, Nissen S, Rosenfeld ME, Wagner WD. AHA Monograph Series. Armonk, NY: Futura Publishing; 1998.

4. Yuan C, Mitsumori LM, Beach KW, Maravilla KR: Special review: Carotid atherosclerotic plaque: noninvasive MR characterization and identification of vulnerable lesions. Radiology 200I, 221:285-99.

5. Saam T, Ferguson MS, Yarnykh VL, Takaya N, Xu D, Polissar NL, Hatsukami TS, Yuan C: Quantitative evaluation of carotid plaque composition by in vivo MRI. Arterioscler Thromb Vasc Biol 2005, 25(I):234-9.

6. Yuan C, Mitsumori LM, Ferguson MS, Polissar NL, Echelard DE, Ortiz G, Small R, Davies JW, Kerwin WS, Hatsukami TS: In vivo accuracy of multispectral MR imaging for identifying lipid-rich necrotic cores and intraplaque hemorrhage in advanced human carotid plaques. Circulation 200I, 104:205I-2056.

7. Chandran KB, Mun JH, Choi KK, Chen JS, Hamilton A, Nagaraj A, McPherson DD: A method for in-vivo analysis for regional arterial wall material property alterations with atherosclerosis: preliminary results. Medical Engineering \& Physics 2003, 25:289-298

8. Pedersen PC, Chakareski J, Lara-Montalvo R: Ultrasound characterization of arterial wall structures based on integrated backscatter profiles. Proc for the 2003 SPIE Med Imaging Symposium, San Diego 2003:I I5-I 26.

9. Cai JM, Hatsukami TS, Ferguson MS, Small R, Polissar NL, Yuan C: Classification of human carotid atherosclerotic lesions with in vivo multicontrast magnetic resonance imaging. Circulation 2002, 106: I368-1373.

10. Holzapfel GA, Stadler M, Schulze-Bause CA): A layer-specific three-dimensional model for the simulation of balloon angi- oplasty using Magnetic Resonance Imaging and mechanical testing. Ann Biomed Eng 2002, 30(6):753-767.

II. Steinman DA: Image-based computational fluid dynamics modeling in realistic arterial geometries. Ann Biomed Eng 2002, 30(4):483-97.

12. Tang D, Yang C, Zheng J, Woodard PK, Saffitz JE, Petruccelli JD, Sicard GA, Yuan C: Local maximal stress hypothesis and computational plaque vulnerability index for atherosclerotic plaque assessment. Ann Biomed Eng 2005, 33( ( 2): I789-I80I.

13. Tang D, Yang C, Mondal S, Liu F, Canton G, Hatsukami TS, Yuan C: A negative correlation between human carotid atherosclerotic plaque progression and plaque wall stress: in vivo MRIBased 2D/3D FSI models. J Biomechanics 2008, 4I(4):727-736.

14. Tang D, Yang C, Kobayashi S, Ku DN: Effect of a lipid pool on stress/strain distributions in stenotic arteries: 3D FSI models. J Biomechanical Engineering 2004, I 26:363-370.

15. Tang D, Yang C, Zheng J, Woodard PK, Sicard GA, Saffitz JE, Yuan C: 3D MRI-based multi-component FSI models for atherosclerotic plaques: a 3-D FSI model. Annals of Biomedical Engineering 2004, 32(7):947-960.

16. Vengrenyuk Y, Carlier S, Xanthos S, Cardoso L, Ganatos P, Virmani $R$, Einav S, Gilchrist L, Weinbaum S: A hypothesis for vulnerable plaque rupture due to stress-induced debonding around cellular microcalcifications in thin fibrous caps. Proc Natl Acad Sci USA 2006, I 03(40): I 4678-83.

17. Bluestein D, Alemu Y, Avrahami I, Gharib M, Dumont K, Ricotta JJ, Einav S: Influence of microcalcifications on vulnerable plaque mechanics using FSI modeling. J Biomech 2008, 4I(5): IIII-I II8.

18. Kaazempur-Mofrad MR, Isasi AG, Younis HF, Chan RC, Hinton DP, Sukhova G, Lamuraglia GM, Lee RT, Kamm RD: Characterization of the atherosclerotic carotid bifurcation using MRI, finite element modeling, and histology. Annals of Biomedical Engineering 2004, 32(7):932-946.

19. Li ZY, Howarth SP, Tang T, Graves MJ, U-King-Im J, Trivedi RA, Kirkpatrick PJ, Gillard JH: Structural analysis and magnetic resonance imaging predict plaque vulnerability: a study comparing symptomatic and asymptomatic individuals. J Vasc Surg 2007, 45(4):768-75.

20. Long $Q, X u X Y$, Ariff $B$, Thom $S A$, Hughes $A D$, Stanton AV: Reconstruction of blood flow patterns in a human carotid bifurcation: A combined CFD and MRI study. J Magn Reson Imaging 2000, II:299-3II.

21. Groen HC, Gijsen FJ, Lugt A van der, Ferguson MS, Hatsukami TS, Steen AF van der, Yuan C, Wentzel J]: Plaque rupture in the carotid artery is localized at the high shear stress region: a case report. Stroke 2007, 38:2379-2381.

22. Loree HM, Kamm RD, Stringfellow RG, Lee RT: Effects of fibrous cap thickness on peak circumferential stress in model atherosclerotic vessels. Circ Res 1992, 71:850-8.

23. Williamson SD, Lam $Y$, Younis HF, Huang $H$, Patel $S$, Kaazempur-Mof$\mathrm{rad}$ MR, Kamm RD: On the sensitivity of wall stresses in diseased arteries to variable material properties. J Biomechanical Engineering 2003, I 25: I47-I55.

24. Yang C, Tang D, Yuan C, Hatsukami TS, Zheng J, Woodard PK: In vivolex vivo MRI-based 3D models with fluid-structure interactions for human atherosclerotic plaques compared with fluid/wall-only models. CMES: Computer Modeling in Engineering and Sciences 2007, 1 9(3):233-245.

25. Kerwin W, Xu D, Liu F, Saam T, Underhill H, Takaya N, Chu B, Hatsukami T, Yuan C: Magnetic resonance imaging of carotid atherosclerosis: Plaque analysis. Topics in Magnetic Resonance Imaging 2007, 18:37I-378.

26. Bathe KJ: Theory and Modeling Guide Vol I: ADINA; Vol II: ADINA-F, ADINA R\&D, Inc., Watertown, MA; 2002. 